\title{
EXPONENTIAL STABILITY OF MILD SOLUTIONS OF STOCHASTIC PARTIAL DIFFERENTIAL EQUATIONS WITH DELAYS
}

\author{
Tomás Caraballo \\ Dpto. Ecuaciones Diferenciales y Análisis Numérico. \\ Universidad de Sevilla. Apartado de Correos 1160. \\ 41080-SEVILLA (Spain) \\ e-mail: caraball@numer.us.es \\ Kai Liu \\ Department of Statistics and Modelling Science \\ University of Strathclyde \\ Glasgow G1 1XH, Scotland, UK \\ e-mail: kai@stams.strath.ac.uk
}

\begin{abstract}
A semilinear stochastic partial differential equation with variable delays is considered. Sufficient conditions for the exponential stability in the $p$-th mean of mild solutions are obtained. Also, pathwise exponential stability is proved. Since the technique of Lyapunov functions is not suitable for delayed equations, the results have been proved by using the properties of the stochastic convolution. As the sufficient conditions obtained are also valid for the case without delays, one can ensure exponential stability of mild solution in some cases where the sufficient conditions in Ichikawa [11] do not give any answer. The results are illustrated with some examples.
\end{abstract}

\section{INTRODUCTION}

The qualitative properties of solutions of stochastic differential equations in infinite dimensions have been investigated by many authors (see, for example, Ichikawa [11], Haussmann [10], Curtain [6], Chow [5], Mao [15], Zabczyk [17, 18], Caraballo [1, 2], Caraballo and Real $[3,4], \ldots$, among others) because of the importance in applications. Some of such researches have considered delayed equations (see Real [16], Caraballo [1, 2], Caraballo and Real [3], Mao [15], Ladde et al. [14],...)

As it is well known, in the case without delays, Lyapunov's technique is available to obtain sufficient conditions for the stability of solutions. However, in the 
case of differential equations with retarded arguments, even with constant delays, Lyapunov's method is not suitable as Krasovskii [12] pointed out for the ordinary differential equations, and Kushner [13] and El'sgol'ts and Norkin [9] (among others) also did for the stochastic differential equations, since the history of the process must be taken into account. For this reason, it is developed in [2] a technique in order to obtain sufficient conditions for the exponential stability of the strong solutions of a linear stochastic partial differential equation with delays. Nevertheless, this method can not be used to analyze the stability of mild solutions since it requires Ito's formula and this does not hold for mild solutions. Ichikawa in [11] solved this problem, for a semilinear stochastic evolution equation, by introducing approximating systems with strong solutions and using a limiting argument. He obtained sufficient conditions for the stability of moments and sample paths in terms of similar functions to Lyapunov ones. But, unfortunately, when we consider a version of the semilinear stochastic equation in [11] with deviating arguments, we encounter a difficulty in order to construct Lyapunov functions which enable us to ensure the exponential stability of mild solutions. However, we have solved this problem by using some properties of the stochastic convolution (see Da Prato and Zabczyk [7]).

In this paper, we consider a semilinear stochastic evolution equation with delays

$$
\left\{\begin{array}{l}
d x(t)=[A x(t)+B(x(\rho(t)))] d t+C(x(\tau(t))) d w(t) \\
x(t)=\psi(t), \quad t \in[-h, 0]
\end{array}\right.
$$

where $A$ is a closed linear operator generating the strongly continuous semigroup $S_{t}$ on the separable Hilbert space $H, w(t)$ is a Wiener process on the separable Hilbert space $U$ with covariance operator $Q \in \mathcal{L}(U, U), B: H \rightarrow H$ and $C: H \rightarrow \mathcal{L}(U, H)$ are Lipschitz continuous, $\rho, \tau:[0,+\infty) \rightarrow[-h, \infty)(h \geq 0)$ are suitable delay functions (see Section 2 for details), and $\psi:[-h, 0] \times \Omega \rightarrow H$ is the initial datum.

Once the existence of mild solutions of (1) is guaranteed, we shall establish the exponential asymptotic stability in the $p$-th mean of the mild solutions. Futhermore, by discussing almost sure estimates of any mild solution of (1), we shall consider almost sure asymptotic behavior of any mild solution.

The results we prove in this paper allow us to ensure exponential stability of moments and paths in some cases where Ichikawa's ones do not give any answer, since our results can also be applied in the case without delays. Finally, we illustrate our theory with two examples.

\section{PRELIMINARIES}

Let $U$ and $H$ be separable Hilbert spaces and let $\mathcal{L}(U, H)$ be the space of all bounded linear operators from $U$ to $H$. We denote the norms of elements in $H, U$ and $\mathcal{L}(U, H)$ by symbols $\|\cdot\|_{H},\|\cdot\|_{U}$ and $\|\cdot\|_{\mathcal{L}(U, H)}$ respectively. But, we will use $\|\cdot\|$ when there is no doubt about the space. We are given a $Q$-Wiener 
process in the complete probability space $\left(\Omega, \mathcal{F}, P,\left\{\mathcal{F}_{t}\right\}_{t \geq 0}\right)$ and having values in $U$, i.e. (see [7]) $w(t)$ is defined as

$$
w(t)=\sum_{n=1}^{\infty} \sqrt{\lambda_{n}} \beta_{n}(t) e_{n}, \quad t \geq 0,
$$

where $\beta_{n}(t)(n=1,2,3, \ldots)$ is a sequence of real valued standard Brownian motions mutually independent on $\left(\Omega, \mathcal{F}, P,\left\{\mathcal{F}_{t}\right\}_{t \geq 0}\right), \lambda_{n} \geq 0(n=1,2,3, \ldots)$ are nonnegative real numbers such that $\sum_{n>1} \lambda_{n}<\infty,\left\{e_{n}\right\}_{n \geq 1}$ is a complete orthonormal basis in $U$, and $Q \in \mathcal{L}(U, U)$ is the incremental covariance operator of the process $w(t)$, which is a symmetric nonnegative trace class operator defined by

$$
Q e_{n}=\lambda_{n} e_{n}, \quad n=1,2,3, \ldots
$$

Now, we need the definition of the $H$-valued stochastic integral with respecto to the $U$-valued $Q$-Wiener process $w(t)$. In the construction of such integral an important rôle will be played by the space of all Hilbert-Schmidt operators $L_{2}^{0}=L_{2}\left(U_{0}, H\right)$ from $U_{0}=Q^{1 / 2}(U)$ into $H$ which is a separable Hilbert space, equipped with the norm

$$
\|\Psi\|_{2}^{2}=\operatorname{Tr}\left(\Psi Q \Psi^{*}\right) .
$$

Let $\Phi:(0, \infty) \rightarrow L_{2}^{0}$ be a predictable, $\mathcal{F}_{t}$-adapted process such that

$$
\int_{0}^{t} E\|\Phi(s)\|_{2}^{2} d s<\infty, \quad \forall t>0,
$$

then, we can define the $H$-valued stochastic integral

$$
\int_{0}^{t} \Phi(s) d w(s)
$$

which will be a continuous square integrable martingale. For that construction, see Da Prato and Zabczyk [7].

In order to set our problem, we consider a linear closed operator $A$ generating a strongly continuous semigroup $S_{t}$ on $H$ and such that

$$
\left\|S_{t}\right\| \leq M e^{-\gamma t} \quad \forall t \geq 0, \quad \text { where } \quad M \geq 1 \quad \text { and } \quad \gamma>0 .
$$

Let $B: H \rightarrow H$ and $C: H \rightarrow \mathcal{L}(U, H)$ be Lipschitz continuous, i.e. there exist positive constants $L_{1}, L_{2}$ such that for any $x, y \in H$ the following conditions are satisfied

$$
\begin{aligned}
\|B(x)-B(y)\|_{H} & \leq L_{1}\|x-y\|_{H} \\
\|C(x)-C(y)\|_{2} & \leq L_{2}\|x-y\|_{H}
\end{aligned}
$$


We are given two continuously differentiable functions of delay $\rho, \tau:[0, \infty) \rightarrow[-h, \infty)$ where $h>0$ and we assume that

$$
\rho^{\prime}(t) \geq 1, \tau^{\prime}(t) \geq 1,-h \leq \rho(t) \leq t,-h \leq \tau(t) \leq t \quad \forall t \geq 0
$$

(Note that the functions $\rho(t)=t-h_{1}, \tau(t)=t-h_{2}$ with $h_{1}, h_{2}>0$ satisfy the precedent hypotheses by setting $\left.h=\max \left\{h_{1}, h_{2}\right\}\right)$.

Remark. We observe that there exists a constant $k \geq 0$ such that

$$
\rho^{-1}(t) \leq t+k, \quad \tau^{-1}(t) \leq t+k, \quad \forall t \geq-h .
$$

And finally, we consider an initial datum $\psi:[-h, 0] \times \Omega \rightarrow H$ such that $\psi(t)$ is $\mathcal{F}_{0}$-measurable for all $t \in[-h, 0], E\|\psi(0)\|^{2}<\infty$ and $\int_{-h}^{0} E\|\psi(s)\|^{2} d s<\infty$.

Since we are mainly interested in the stability properties of mild solutions, we will consider the following equation instead of (1)

$$
\begin{aligned}
& x(t)=S_{t} \psi(0)+\int_{0}^{t} S_{t-s} B(x(\rho(s))) d s+\int_{0}^{t} S_{t-s} C(x(\tau(s))) d w(s), t \geq 0 \\
& x(t)=\psi(t), \quad t \in[-h, 0]
\end{aligned}
$$

and we will assume that for each initial value $\psi$ and each $T>0$ there exists a unique mild solution of (1), i.e. there exists a process $x^{\psi}(t)(t \in[-h, T])$ which is $H$-valued, $\mathcal{F}_{t}$-adapted (with $\mathcal{F}_{t}=\mathcal{F}_{0}$ for $t \in[-h, 0]$ ) and predictable with $\int_{-h}^{T}\left\|x^{\psi}(t)\right\|^{2} d t<\infty$ w.p.1, and satisfies (12) and (13) w.p.1. (A result about the existence and uniqueness of mild solutions is sketched in the Appendix).

Definition 2.1 Let $p \geq 2$ be an integer. The mild solution $x^{\psi}(t)$ of (1) (with $E\|\psi(0)\|^{p}<\infty$ and $\left.\int_{-h}^{0} E\|\psi(s)\|^{p} d s<\infty\right)$ is said to be globally exponentially asymptotically stable in the $p$-th mean if there exist $a>0$ and $L \geq 1$ such that, for any mild solution of (1) $x^{\phi}(t)$ corresponding to an initial value $\phi$ with $E\|\phi(0)\|^{p}<\infty, \int_{-h}^{0} E\|\phi(s)\|^{p} d s<\infty$, the following inequality is satisfied:

$$
E\left\|x^{\psi}(t)-x^{\phi}(t)\right\|_{H}^{p} \leq L e^{-a t}\|\psi-\phi\|_{1}^{p}, \quad t \geq 0,
$$

where $\|\psi-\phi\|_{1}^{p}=\max \left\{E\|\psi(0)-\phi(0)\|^{p}, \int_{-h}^{0} E\|\psi(s)-\phi(s)\|^{p} d s\right\}$.

\section{EXPONENTIAL STABILITY IN THE $p$-TH MEAN}

In this section we shall discuss the exponential asymptotic stability in the $p$-th mean of mild solutions to (1).

THEOREM 3.1 Let $p \geq 2$ be an integer and let $x(t)=x^{\psi}(t)$ and $y(t)=x^{\phi}(t)$ be solutions of (12),(13) with initial values $\psi$ and $\phi$ respectively. Assume that conditions (7), (8), (9) and (10) are satisfied. Then, the following inequality holds:

$$
E\|x(t)-y(t)\|_{H}^{p} \leq \beta\|\psi-\phi\|_{1}^{p} e^{-(\gamma-\alpha) t}, \quad t \geq 0,
$$


where $\alpha=3^{p-1} M^{p}\left(\sigma_{1}+\sigma_{2}\right), \beta=3^{p-1} M^{p}\left(1+\sigma_{1}+\sigma_{2}\right), \sigma_{1}=\gamma^{1-p} L_{1}^{p} e^{\gamma k}$, $\sigma_{2}=c_{p} L_{2}^{p} e^{\gamma k}(2 \gamma(p-1) /(p-2))^{1-p / 2}$ and $c_{p}=(p(p-1) / 2)^{p / 2}$.

Proof. Since $x(t)$ and $y(t)$ are two solutions of (12), we have

$$
\begin{aligned}
& x(t)=S_{t} \psi(0)+\int_{0}^{t} S_{t-s} B(x(\rho(s))) d s+\int_{0}^{t} S_{t-s} C(x(\tau(s))) d w(s), \quad t \geq 0 \\
& y(t)=S_{t} \phi(0)+\int_{0}^{t} S_{t-s} B(y(\rho(s))) d s+\int_{0}^{t} S_{t-s} C(y(\tau(s))) d w(s), \quad t \geq 0 .
\end{aligned}
$$

Thus, it follows that

$$
\begin{aligned}
E\|x(t)-y(t)\|^{p} \leq & 3^{p-1} E\left\|S_{t}(\psi(0)-\phi(0))\right\|_{H}^{p} \\
& +3^{p-1} E\left\|\int_{0}^{t} S_{t-s}(B(x(\rho(t)))-B(y(\rho(t)))) d s\right\|_{H}^{p} \\
& +3^{p-1} E\left\|\int_{0}^{t} S_{t-s}(C(x(\tau(t)))-C(y(\tau(t)))) d w(s)\right\|_{H}^{p} .
\end{aligned}
$$

Now, we estimate the terms on the right-hand side of (15).

Firstly, by condition (7), we obtain

$$
E\left\|S_{t}(\psi(0)-\phi(0))\right\|_{H}^{p} \leq M^{p} e^{-p \gamma t} E\|\psi(0)-\phi(0)\|_{H}^{p} \leq M^{p} e^{-p \gamma t}\|\psi-\phi\|_{1}^{p}
$$

Secondly, Holder's inequality, conditions (8), (7) and the change of variables $u=$ $\rho(s)$ yield

$$
\begin{aligned}
& E\left\|\int_{0}^{t} S_{t-s}(B(x(\rho(s)))-B(y(\rho(s)))) d s\right\|_{H}^{p} \\
\leq & E\left[\int_{0}^{t}\left\|S_{t-s}\right\|\|B(x(\rho(s)))-B(y(\rho(s)))\|_{H} d s\right] \\
\leq & E\left[\int_{0}^{t} M e^{-\gamma(t-s)}\|B(x(\rho(s)))-B(y(\rho(s)))\|_{H} d s\right]^{p} \\
\leq & M^{p} E\left[\int_{0}^{t} e^{-(\gamma(p-1) / p)(t-s)} e^{-(\gamma / p)(t-s)}\|B(x(\rho(s)))-B(y(\rho(s)))\|_{H} d s\right]^{p} \\
\leq & M^{p}\left[\int_{0}^{t} e^{-\gamma(t-s)} d s\right]^{p-1} \int_{0}^{t} e^{-\gamma(t-s)} E\|B(x(\rho(s)))-B(y(\rho(s)))\|_{H}^{p} d s \\
\leq & M^{p}(1 / \gamma)^{p-1} L_{1}^{p} \int_{0}^{t} e^{-\gamma(t-s)} E\|x(\rho(s))-y(\rho(s))\|_{H}^{p} d s \\
\leq & M^{p}(1 / \gamma)^{p-1} L_{1}^{p} \int_{\rho(0)}^{\rho(t)} e^{-\gamma\left(t-\rho^{-1}(u)\right)} E\|x(u)-y(u)\|_{H}^{p} \frac{d u}{\rho^{\prime}\left(\rho^{-1}(u)\right)} \\
\leq & M^{p}(1 / \gamma)^{p-1} L_{1}^{p} \int_{-h}^{t} e^{-\gamma(t-u-k)} E\|x(u)-y(u)\|_{H}^{p} d u
\end{aligned}
$$




$$
\begin{aligned}
\leq \quad & M^{p}(1 / \gamma)^{p-1} L_{1}^{p} e^{\gamma k} \int_{-h}^{0} e^{-\gamma(t-u)} E\|x(u)-y(u)\|_{H}^{p} d u \\
& +M^{p}(1 / \gamma)^{p-1} L_{1}^{p} e^{\gamma k} \int_{0}^{t} e^{-\gamma(t-u)} E\|x(u)-y(u)\|_{H}^{p} d u \\
\leq \quad & M^{p}(1 / \gamma)^{p-1} L_{1}^{p} e^{\gamma k} \int_{-h}^{0} e^{-\gamma(t-u)} E\|\psi(u)-\phi(u)\|_{H}^{p} d u \\
& \quad+M^{p}(1 / \gamma)^{p-1} L_{1}^{p} e^{\gamma k} \int_{0}^{t} e^{-\gamma(t-u)} E\|x(u)-y(u)\|_{H}^{p} d u \\
\leq \quad & M^{p}(1 / \gamma)^{p-1} L_{1}^{p} e^{\gamma k} e^{-\gamma t}\|\psi-\phi\|_{1}^{p} \\
& +M^{p}(1 / \gamma)^{p-1} L_{1}^{p} e^{\gamma k} \int_{0}^{t} e^{-\gamma(t-s)} E\|x(s)-y(s)\|_{H}^{p} d s \\
\leq \quad M^{p} \sigma_{1} e^{-\gamma t}\|\psi-\phi\|_{1}^{p} & +M^{p} \sigma_{1} \int_{0}^{t} e^{-\gamma(t-s)} E\|x(s)-y(s)\|_{H}^{p} d s,
\end{aligned}
$$

where $\sigma_{1}=\gamma^{1-p} L_{1}^{p} e^{\gamma k}$

As for the third term on the right-hand side of (15), and in addition to Holder's inequality, conditions (9), (7) and the change of variables $u=\tau(s)$, the following inequality (see Lemma 7.7 from [7]) will be used:

$$
E\left\|\int_{0}^{t} \Phi(s) d w(s)\right\|_{H}^{p} \leq c_{p}\left(\int_{0}^{t}\left(E\|\Phi(s)\|_{2}^{p}\right)^{2 / p} d s\right)^{p / 2}
$$

for an arbitrary $L_{2}^{0}$-predictable process $\Phi$ and $p \geq 2$, where $c_{p}=(p(p-1) / 2)^{p / 2}$. So, if $p>2$ we can deduce

$$
\begin{aligned}
E & \left\|\int_{0}^{t} S_{t-s}(C(x(\tau(s)))-C(y(\tau(s)))) d w(s)\right\|_{H}^{p} \\
& \leq c_{p} M^{p}\left\{\int_{0}^{t}\left(e^{-\gamma p(t-s)} E\|C(x(\tau(s)))-C(y(\tau(s)))\|_{2}^{p}\right)^{2 / p} d s\right\}^{p / 2} \\
& \leq c_{p} M^{p} L_{2}^{p}\left\{\int_{0}^{t}\left(e^{-\gamma p(t-s)} E\|x(\tau(s))-y(\tau(s))\|_{H}^{p}\right)^{2 / p} d s\right\}^{p / 2} \\
& \leq c_{p} M^{p} L_{2}^{p}\left\{\int_{0}^{t}\left(e^{-\gamma(p-1)(t-s)} e^{-\gamma(t-s)} E\|x(\tau(s))-y(\tau(s))\|_{H}^{p}\right)^{2 / p} d s\right\}^{p / 2} \\
& \leq c_{p} M^{p} L_{2}^{p}\left\{\int_{0}^{t} e^{-\left[\frac{2(p-1)}{p-2}\right] \gamma(t-s)} d s\right\}^{\frac{p}{2}-1} \int_{0}^{t} e^{-\gamma(t-s)} E\|x(\tau(s))-y(\tau(s))\|_{H}^{p} d s \\
& \leq c_{p} M^{p} L_{2}^{p}(2 \gamma(p-1) /(p-2))^{1-p / 2} \int_{0}^{t} e^{-\gamma(t-s)} E\|x(\tau(s))-y(\tau(s))\|_{H}^{p} d s . \quad(19)
\end{aligned}
$$


We remark that if $p=2$, then inequality (19) also holds with $0^{0}:=1$. Consequently, proceeding as we did previously, we can obtain for $p \geq 2$,

$$
\begin{aligned}
& E\left\|\int_{0}^{t} S_{t-s}(C(x(\tau(s)))-C(y(\tau(s)))) d w(s)\right\|_{H}^{p} \\
& \leq c_{p} M^{p} L_{2}^{p}(2 \gamma(p-1) /(p-2))^{1-p / 2} \int_{0}^{t} e^{-\gamma(t-s)} E\|x(\tau(s))-y(\tau(s))\|_{H}^{p} d s \\
& \quad \leq M^{p} \sigma_{2}\left[e^{-\gamma t}\|\psi-\phi\|_{1}^{p}+\int_{0}^{t} e^{-\gamma(t-s)} E\|x(s)-y(s)\|_{H}^{p} d s\right],
\end{aligned}
$$

where $\sigma_{2}=c_{p} L_{2}^{p} e^{\gamma k}(2 \gamma(p-1) /(p-2))^{1-p / 2}$.

¿From (15)-(20), it follows

$$
\begin{aligned}
& E\|x(t)-y(t)\|^{p} \\
& \leq \quad 3^{p-1} M^{p}\left(1+\sigma_{1}+\sigma_{2}\right)\|\psi-\phi\|_{1}^{p} e^{-\gamma t} \\
& \quad+3^{p-1} M^{p}\left(\sigma_{1}+\sigma_{2}\right) \int_{0}^{t} e^{-\gamma(t-s)} E\|x(s)-y(s)\|_{H}^{p} d s \quad \forall t \geq 0,
\end{aligned}
$$

and,

$$
\begin{aligned}
e^{\gamma t} E\|x(t)-y(t)\|^{p} & \leq 3^{p-1} M^{p}\left(1+\sigma_{1}+\sigma_{2}\right)\|\psi-\phi\|_{1}^{p} \\
& +3^{p-1} M^{p}\left(\sigma_{1}+\sigma_{2}\right) \int_{0}^{t} e^{\gamma s} E\|x(s)-y(s)\|_{H}^{p} d s .
\end{aligned}
$$

Finally, Gronwall's inequality yields

$$
\begin{aligned}
& e^{\gamma t} E\|x(t)-y(t)\|^{p} \leq 3^{p-1} M^{p}\left(1+\sigma_{1}+\sigma_{2}\right)\|\psi-\phi\|_{1}^{p} e^{3^{p-1} M^{p}\left(\sigma_{1}+\sigma_{2}\right) t}, \\
& E\|x(t)-y(t)\|^{p} \leq 3^{p-1} M^{p}\left(1+\sigma_{1}+\sigma_{2}\right)\|\psi-\phi\|_{1}^{p} e^{-(\gamma-\alpha) t}, \quad \forall t \geq 0,
\end{aligned}
$$

where $\alpha=3^{p-1} M^{p}\left(\sigma_{1}+\sigma_{2}\right)$, which completes the proof.

Remark. Observe that the constant $\alpha$ in Theorem 3.1 depends on $M, \gamma, L_{1}, L_{2}$ and $k$. Therefore, if the problem we are dealing with is such that $\alpha\left(M, \gamma, L_{1}, L_{2}, k\right)<$ $\gamma$, then we can assure that all mild solutions to this problem are exponentially asymptotically stable in the $p$-th mean. But, what does $\alpha<\gamma$ mean? Let us look at the following cases:

- Case 1: We note that problem (1) can be interpreted as a perturbed problem of the following linear one:

$$
\left\{\begin{array}{l}
d x(t)=A x(t) d t, \quad t>0 \\
x(t)=\psi(t), \quad t \in[-h, 0]
\end{array}\right.
$$

Of course, hypothesis (7) tells us that solutions to (24) are exponentially asymptotically stable. Consequently, what it is proved in the previous theorem is the following: 
If the unperturbed (linear) problem (24) is exponentially asymptotically stable and the perturbations appearing in (1) are small enough (in the sense that $\alpha<$ $\gamma$ ), then all mild solutions to the perturbed problem (1) are also exponentially asymptotically stable in the $p$-th mean (that is, a first approximation result on exponential stability holds).

Thus, given the linear problem (24) where the semigroup is exponentially stable (i.e. hypothesis (7) is satisfied), condition $\alpha<\gamma$ represents how large $B$ and $C$ can be (or what it is the same, how large $L_{1}$ and $L_{2}$ are permitted to be) so that the mild solutions to the perturbed problem (1) remain exponentially stable in the $p$-th mean.

In addition to this, note that condition $\alpha<\gamma$ means

$$
\frac{L_{1}^{p}}{3^{1-p} M^{-p} e^{-\gamma k} \gamma^{p}}+\frac{L_{2}^{p}}{3^{1-p} M^{-p} e^{-\gamma k}(p-2 / p-1)^{p / 2-1} c_{p}^{-1} \gamma^{p / 2}}<1 .
$$

Therefore, if the delayed Lipschitz perturbations are such that $\left(L_{1}, L_{2}\right)$ is inside the region in the $X_{1} X_{2}$-plane delimited by the positive semi-axes and the curve given by (25) (replacing $L_{1}$ and $L_{2}$ by the coordinates $x_{1}$ and $x_{2}$ and $<$ by $=$ ), then the exponential stability of mild solutions follows. Observe that, when $p=2$, such a curve is an ellipse whose semi-axes only depend on the semigroup constants $M$ and $\gamma$, and on $k$ (the constant related to the delay functions), and are given by

$$
3^{-1 / 2} M^{-1} e^{-\gamma k / 2} \gamma, \quad 3^{-1 / 2} M^{-1} e^{-\gamma k / 2} \gamma^{1 / 2} .
$$

- Case 2: Another question we can consider is the following: What is the minimum value of $\gamma$ which guarantees exponential stability of mild solutions? In other words, what is the minimum value of $\gamma$ satisfying $\alpha<\gamma$ ? As we shall illustrate immediately, this value can exist or not depending on the smallness of the Lipschitz constants.

Assume, for simplicity, $p=2, M=1, k=1$. Now, condition $\alpha<\gamma$ turns to

$$
3 e^{\gamma}\left(\frac{L_{1}^{2}}{\gamma^{2}}+\frac{L_{2}^{2}}{\gamma}\right)<1 .
$$

So, if we want to find the minimum value of $\gamma$ such that the last inequality holds, we must look for the minimum positive value of $\gamma$, denoted $\gamma^{*}$, such that $f(\gamma)<0$, where $f$ is the real function given by

$$
f(\gamma)=3 e^{\gamma}\left(\frac{L_{1}^{2}}{\gamma^{2}}+\frac{L_{2}^{2}}{\gamma}\right)-1 .
$$

By studying this function, we obtain the following: 
a) There exists a unique absolute minimum of $f$ in the interval $(0,+\infty)$, denoted by $\gamma_{+}$;

b) $f$ is strictly decreasing in the interval $\left(0, \gamma_{+}\right)$and strictly increasing in $\left(\gamma_{+},+\infty\right)$, where $\gamma_{+}$is now given by

$$
\gamma_{+}=\frac{L_{2}^{2}-L_{1}^{2}+\sqrt{L_{1}^{4}+L_{2}^{4}+6 L_{1}^{2} L_{2}^{2}}}{2 L_{2}^{2}} \quad\left(L_{2} \neq 0\right) .
$$

So, there are two possibilities:

Possibility 1: $f\left(\gamma_{+}\right) \geq 0$, which implies that $\gamma^{*}$ does not exist.

Possibility 2: $f\left(\gamma_{+}\right)<0$, and then $\gamma^{*}$ is the unique zero of $f$ in $\left(0, \gamma_{+}\right)$.

But, in order to ensure that possibility 2 holds, the constants $L_{1}$ and $L_{2}$ must be small enough. Indeed, if we assume for simplicity that $L_{1}=L_{2}=a$, we get that $\gamma_{+}=\sqrt{2}$. So, $f(\sqrt{2})<0$ means

$$
a^{2}<2(1+\sqrt{2})^{-1} 3^{-\sqrt{2}}
$$

Consequently, we can state the following theorem as a corollary of Theorem 3.1

TheOREM 3.2 Assume the hypotheses of Theorem 3.1 with $\gamma>\alpha$. Then, all mild solutions of (1) are globally exponentially asymptotically stable in the $p$-th mean.

\section{ALMOST SURE ASYMPTOTIC BEHAVIOUR}

In this section, we state the pathwise asymptotic stability for the mild solutions of (1) by using a technique close to Haussmann's [10](see also [11], [2]). We shall split our study in two cases because of the properties of the stochastic convolution. Firstly, we recall two useful lemmas on such convolution which were proved by Da Prato and Zabczyk.

Lemma 4.1 (See Da Prato and Zabczyk [8], p.144) Let $\left\|S_{t}\right\| \leq M$ for all $t \geq 0$ and let $\Phi:[0, \infty) \rightarrow L_{2}^{0}$ be a predictable, $\mathcal{F}_{t}$-adapted process with $\int_{0}^{t} E\|\Phi(s)\|_{2}^{p} d s<\infty$ for some integer $p>2$ and any $t \geq 0$. Then there exists a constant $k_{p}>0$ such that for any natural number $N$

$$
E\left\{\sup _{N \leq t \leq N+1}\left\|\int_{N}^{t} S_{t-s} \Phi(s) d w(s)\right\|_{H}^{p}\right\} \leq k_{p} \int_{N}^{N+1} E\|\Phi(s)\|_{2}^{p} d s .
$$

Lemma 4.2 (See Da Prato and Zabczyk [7], p.160) Assume that A generates a strongly continuous contraction semigroup. Let $\Phi:[0, \infty) \rightarrow L_{2}^{0}$ be a predictable, 
$\mathcal{F}_{t}$-adapted process such that $\int_{0}^{t} E\|\Phi(s)\|_{2}^{2} d s<\infty$ for any $t \geq 0$. Then there exists a constant $k_{2}>0$ such that for any natural number $N$

$$
E\left\{\sup _{N \leq t \leq N+1}\left\|\int_{N}^{t} S_{t-s} \Phi(s) d w(s)\right\|_{H}^{2}\right\} \leq k_{2} \int_{N}^{N+1} E\|\Phi(s)\|_{2}^{2} d s .
$$

Now, we can consider almost sure asymptotic behavior of solutions.

TheOrem 4.1 Suppose that all conditions of Theorem 3.1 hold with $p>2$, and let $x(t)$ and $y(t)$ be solutions of (12), (13) with initial data $\psi$ and $\phi$ respectively. If $\gamma>\alpha$, then there exists $T(\omega)>0$ such that for all $t \geq T(\omega)$

$$
\|x(t)-y(t)\|_{H}^{p} \leq \delta\|\psi-\phi\|_{1}^{p} e^{-(\gamma-\alpha) t / 2} \quad \text { w.p.1, }
$$

where $\delta=e^{(\gamma-\alpha) / 2}$.

Proof. Let $N_{0}$ be an integer such that $\rho\left(N_{0}\right) \geq 0, \tau\left(N_{0}\right) \geq 0$. Since $\rho^{\prime} \geq 1, \tau^{\prime} \geq 1$, it follows that $\rho(N)>0$ and $\tau(N)>0$ for all natural number $N>N_{0}$. Let $N>N_{0}$ and let $I_{N}$ denote the interval $[N, N+1]$. Then, for $t \in I_{N}$, we have

$$
\begin{aligned}
& x(t)=S_{t-N} x(N)+\int_{N}^{t} S_{t-s} B(x(\rho(s))) d s+\int_{N}^{t} S_{t-s} C(x(\tau(s))) d w(s) \\
& y(t)=S_{t-N} y(N)+\int_{N}^{t} S_{t-s} B(y(\rho(s))) d s+\int_{N}^{t} S_{t-s} C(y(\tau(s))) d w(s)
\end{aligned}
$$

and, so

$$
\begin{aligned}
\|x(t)-y(t)\|_{H} \leq \| & S_{t-N}(x(N)-y(N)) \|_{H} \\
& +\left\|\int_{N}^{t} S_{t-s}(B(x(\rho(s)))-B(y(\rho(s)))) d s\right\|_{H} \\
& +\left\|\int_{N}^{t} S_{t-s}(C(x(\tau(s)))-C(y(\tau(s)))) d w(s)\right\|_{H}
\end{aligned}
$$

Thus, for any fixed $\varepsilon>0$, we obtain

$$
\begin{aligned}
P\left[\sup _{t \in I_{N}} \|\right. & \left.x(t)-y(t) \|_{H}>\varepsilon\right] \\
\leq P & {\left[\sup _{t \in I_{N}}\left\|S_{t-N}(x(N)-y(N))\right\|_{H}>\varepsilon / 3\right] } \\
& +P\left[\sup _{t \in I_{N}}\left\|\int_{N}^{t} S_{t-s}(B(x(\rho(s)))-B(y(\rho(s)))) d s\right\|_{H}>\varepsilon / 3\right] \\
& +P\left[\sup _{t \in I_{N}}\left\|\int_{N}^{t} S_{t-s}(C(x(\tau(s)))-C(y(\tau(s)))) d w(s)\right\|_{H}>\varepsilon / 3\right]
\end{aligned}
$$




$$
\begin{aligned}
\leq & (3 / \varepsilon)^{p} E\left[\sup _{t \in I_{N}}\left\|S_{t-N}(x(N)-y(N))\right\|_{H}^{p}\right] \\
& +(3 / \varepsilon)^{p} E\left[\sup _{t \in I_{N}}\left\|\int_{N}^{t} S_{t-s}(B(x(\rho(s)))-B(y(\rho(s)))) d s\right\|_{H}^{p}\right] \\
& +(3 / \varepsilon)^{p} E\left[\sup _{t \in I_{N}}\left\|\int_{N}^{t} S_{t-s}(C(x(\tau(s)))-C(y(\tau(s)))) d w(s)\right\|_{H}^{p}\right] \\
= & T_{1}+T_{2}+T_{3} .
\end{aligned}
$$

Now, Theorem 3.1 and Holder's inequality yield

$$
\begin{aligned}
T_{1} & =(3 / \varepsilon)^{p} E\left[\sup _{t \in I_{N}}\left\|S_{t-N}(x(N)-y(N))\right\|_{H}^{p}\right] \\
& \leq(3 M / \varepsilon)^{p} E\left[\sup _{t \in I_{N}} e^{-p \gamma(t-N)}\|x(N)-y(N)\|_{H}^{p}\right] \\
& \leq(3 M / \varepsilon)^{p} E\left[\|x(N)-y(N)\|_{H}^{p}\right] \\
& \leq(3 M / \varepsilon)^{p} \beta\|\psi-\phi\|_{1}^{p} e^{-(\gamma-\alpha) N} . \\
T_{2} & =(3 / \varepsilon)^{p} E\left[\sup _{t \in I_{N}}\left\|\int_{N}^{t} S_{t-s}(B(x(\rho(s)))-B(y(\rho(s)))) d s\right\|_{H}^{p}\right] \\
& \leq(3 / \varepsilon)^{p} E\left[\sup _{t \in I_{N}}\left(\int_{N}^{t}\left\|S_{t-s}\right\|\|B(x(\rho(s)))-B(y(\rho(s)))\|_{H} d s\right)^{p}\right] \\
& \leq\left(3 M L_{1} / \varepsilon\right)^{p} E\left[\sup _{t \in I_{N}} \int_{N}^{t}\|x(\rho(s))-y(\rho(s))\|_{H}^{p} d s\right] \\
& \leq\left(3 M L_{1} / \varepsilon\right)^{p} \int_{N}^{N+1} E\|x(\rho(s))-y(\rho(s))\|_{H}^{p} d s \\
& \leq\left(3 M L_{1} / \varepsilon\right)^{p} \int_{\rho(N)}^{\rho(N+1)} E\|x(s)-y(s)\|_{H}^{p} d s \\
& \leq\left(3 M L_{1} / \varepsilon\right)^{p} \beta\|\psi-\phi\|_{1}^{p} \int_{\rho(N)}^{\rho(N+1)} e^{-(\gamma-\alpha) s} d s \\
\leq & \left(3 M L_{1} / \varepsilon\right)^{p}(\beta /(\gamma-\alpha))\|\psi-\phi\|_{1}^{p} e^{-(\gamma-\alpha) \rho(N)} \\
\leq & \left(3 M L_{1} / \varepsilon\right)^{p}(\beta /(\gamma-\alpha))\|\psi-\phi\|_{1}^{p} e^{-(\gamma-\alpha)(N-h)} \\
\leq & \left(3 M L_{1} / \varepsilon\right)^{p}(\beta /(\gamma-\alpha)) e^{(\gamma-\alpha) h}\|\psi-\phi\|_{1}^{p} e^{-(\gamma-\alpha) N} . \\
&
\end{aligned}
$$

Analogously, by using Lemma 4.1

$$
\begin{aligned}
T_{3} & =(3 / \varepsilon)^{p} E\left[\sup _{t \in I_{N}}\left\|\int_{N}^{t} S_{t-s}(C(x(\tau(s)))-C(y(\tau(s)))) d w(s)\right\|_{H}^{p}\right] \\
& \leq\left(3 L_{2} / \varepsilon\right)^{p} k_{p} \int_{N}^{N+1} E\|x(\tau(s))-y(\tau(s))\|_{H}^{p} d s
\end{aligned}
$$




$$
\leq\left(3 L_{2} / \varepsilon\right)^{p}\left(\beta k_{p} /(\gamma-\alpha)\right) e^{(\gamma-\alpha) h}\|\psi-\phi\|_{1}^{p} e^{-(\gamma-\alpha) N}
$$

Consequently,

$$
P\left[\sup _{t \in I_{N}}\|x(t)-y(t)\|_{H}>\varepsilon\right] \leq\left(m / \varepsilon^{p}\right)\|\psi-\phi\|_{1}^{p} e^{-(\gamma-\alpha) N},
$$

where $m=\beta\left((3 M)^{p}\left(1+\left(L_{1}^{p} /(\gamma-\alpha)\right) e^{(\gamma-\alpha) h}+\left(3 L_{2}\right)^{p}\left(k_{p} /(\gamma-\alpha)\right) e^{(\gamma-\alpha) h}\right)\right.$. For each integer $N \geq N_{0}$, we set $\varepsilon_{N}=\|\psi-\phi\|_{1} e^{-(\gamma-\alpha) N /(2 p)}$. Then, it follows

$$
P\left\{\sup _{t \in I_{N}}\|x(t)-y(t)\|_{H}>\|\psi-\phi\|_{1} e^{-(\gamma-\alpha) N /(2 p)}\right\} \leq m e^{-(\gamma-\alpha) N / 2} .
$$

Therefore, Borel-Cantelli's lemma implies that there exists $T(\omega)>0$ such that for all $t>T(\omega)$

$$
\|x(t)-y(t)\|_{H}^{p} \leq \delta\|\psi-\phi\|_{1}^{p} e^{-(\gamma-\alpha) t / 2} \quad \text { w.p.1 }
$$

where $\delta=e^{(\gamma-\alpha) / 2}$.

Next, in a similar way, we can establish the result in the case $p=2$.

TheOREM 4.2 Assume A generates a strongly continuous contraction semigroup. Under the hypotheses of Theorem 3.2 for $p=2$, the same conclusion of Theorem 4.1 holds for $p=2$.

Proof. We proceed as in Theorem 4.1 using Lemma 4.2 instead of Lemma 4.1.

\section{$5 \quad$ EXAMPLES}

In this section, we are going to show a couple of examples in order to illustrate our theory.

Example 1. Consider a one-dimensional rod of length $\pi$ whose ends are maintained at $0^{\circ}$ and whose sides are insulated. Suppose there is an exothermic reaction taking place inside the rod with heat being produced proportionally to the temperature at a previous time $t-h$ (for the sake of simplicity, we assume the delay $h \geq 0$ is constant). Consequently, the temperature in the rod may be modeled to satisfy

$$
\left\{\begin{array}{l}
\frac{\partial u(t, x)}{\partial t}=\frac{\partial^{2} u(t, x)}{\partial x^{2}}+r u(t-h, x), \quad 0<x<\pi, \quad t>0 \\
u(t, 0)=u(t, \pi)=0, \quad t>0, \\
u(t, x)=\psi(t, x), \quad t \in[-h, 0], \quad x \in[0, \pi],
\end{array}\right.
$$

where $r$ depends on the rate of reaction and $\psi:[-h, 0] \times[0, \pi] \rightarrow \mathbf{R}$ is a given function. 
We observe that, when there is no heat production (i.e. $r=0$ ), the problem $(27)$ is exponentially stable since, in this case, the solutions are given by

$$
u(t, x)=\sum_{n=1}^{\infty} a_{n} e^{-n^{2} t} \sin n x,
$$

where $h=0$ and $\psi(0, x)=\sum_{n=1}^{\infty} a_{n} \sin n x$.

If we assume $r=r_{0}=$ constant, then it is not difficult to prove that the solutions to (27) remain exponentially stable provided $r_{0}^{2}<1$ (see Caraballo [1]). We note that, in this example, $\gamma=M=1, C=0, B u=r_{0} u, \rho(t)=t-h, L_{1}=r_{0}, L_{2}=0$ and $k=h$. So, condition $r_{0}^{2}<1$ is similar to the one in the Remark preceding Theorem 3.2. Also, we can interpret the result as follows:

Being the solutions to (27) with $r_{0}=0$ exponentially stable, we can assure that the solutions remain exponentially stable if the heat production is small enough (i.e. if $\left.r_{0}^{2}<1\right)$.

However, it often occurs that the exothermic reaction can be random. In some cases, this can be modelled by writing the term $r u(t-h, x)$ in the form $\left(r_{0}+\right.$ $\left.r_{1} \dot{\beta}(t)\right) u(t-h, x)$, where $\beta(t)$ is a real standard Brownian motion (so, $Q=1, \lambda_{1}=$ $\left.1, \lambda_{n}=0, \quad \forall n \geq 2\right)$.

Thus, (27) can be written as:

$$
\left\{\begin{array}{l}
\frac{\partial u(t, x)}{\partial t}=\frac{\partial^{2} u(t, x)}{\partial x^{2}}+r_{0} u(t-h, x)+r_{1} u(t-h, x) \dot{\beta}(t), 0<x<\pi, t>0 \\
u(t, 0)=u(t, \pi)=0, \quad t>0, \\
u(t, x)=\psi(t, x), \quad t \in[-h, 0], \quad x \in[0, \pi],
\end{array}\right.
$$

and setting $H=L^{2}(0, \pi), U=\mathbf{R}, A$ the operator $A=d^{2} / d x^{2}$ with domain

$$
\mathcal{D}(A)=\left\{y \in H: \frac{d y}{d x}, \frac{d^{2} y}{d x^{2}} \in H, y(0)=y(\pi)=0\right\},
$$

$B(u)=r_{0} u, C(u)=r_{1} u, \rho(t)=\tau(t)=t-h$, the problem can be reformulated as

$$
\left\{\begin{array}{l}
d u(t)=(A u(t)+B(u(\rho(t)))) d t+C(u(\tau(t))) d \beta(t), \quad t>0 \\
u(t)=\psi(t), \quad t \in[-h, 0]
\end{array}\right.
$$

and, in order to apply theorems 3.2, 4.1 and 4.2 we need to check that $\alpha<\gamma$. But, as one can compute immediately, $\gamma=M=1$ (i.e., $A$ generates a strongly continuous contraction semigroup), $L_{1}=r_{0}, L_{2}=r_{1}, k=h$ and, consequently, the hypothesis $\alpha<\gamma$ turns to the following in the case $p=2$ :

$$
3 e^{h}\left(r_{0}^{2}+r_{1}^{2}\right)<1 .
$$

Therefore, if $B$ and $C$ are sufficiently small (i.e., for instance, $3 e^{h}\left(r_{0}^{2}+r_{1}^{2}\right)<1$ ), the mild solutions to (29) are exponentially asymptotically stable in mean square (by 
theorem 3.2) and, by the virtue of theorem 4.2, pathwise exponential stability also holds.

Finally, observe that the same result follows if $B$ and $C$ are assumed to be Lipschitz continuous (with constants $L_{1}$ and $L_{2}$ respectively) instead of linear.

Example 2. Now, we consider the semilinear stochastic heat equation with delays

$$
\left\{\begin{array}{l}
d y(x, t)=\left[\frac{\partial^{2} y(x, t)}{\partial x^{2}}-\frac{y(x, \rho(t))}{1+|y(x, \rho(t))|}\right] d t+\frac{\sigma y(x, \tau(t))}{1+|y(x, \tau(t))|} d \beta(t) \\
y(0, t)=y(1, t)=0 \quad \forall t \geq-h, \quad y(x, t)=\psi(x, t), \quad t \in[-h, 0], x \in[0,1],
\end{array}\right.
$$

where $\beta(t)$ is a real standard Wiener process (as in the previous example) and $\sigma$ is a real number. We take $H=L^{2}(0,1), U=\mathbf{R}, \rho, \tau$ and $\psi$ satisfying the hypotheses from Section 2, $B$ and $C$ defined as

$$
B(y)=-\frac{y}{1+|y|}, \quad C(y)=\frac{\sigma y}{1+|y|},
$$

and $A$ the operator $A=d^{2} / d x^{2}$ with domain

$$
\mathcal{D}(A)=\left\{y \in H: \frac{d y}{d x}, \frac{d^{2} y}{d x^{2}} \in H, y(0)=y(1)=0\right\} .
$$

Then, it is well known that the strongly continuous semigroup $S_{t}$ generated by $A$ satisfies

$$
\left\|S_{t}\right\| \leq e^{-\pi^{2} t}, \quad \forall t \geq 0 .
$$

Therefore, $M=1, \gamma=\pi^{2}$ in (7), $L_{1}=1, L_{2}=\sigma, \sigma_{1}=\left(1 / \pi^{2}\right)^{p-1} e^{\pi^{2} k}$, $\sigma_{2}=c_{p} \sigma^{p}\left(2 \pi^{2}(p-1) /(p-2)\right)^{1-p / 2} e^{\pi^{2} k}, \quad c_{p}=(p(p-1) / 2)^{p / 2}, \alpha=3^{p-1}\left(\sigma_{1}+\sigma_{2}\right)$.

Consequently, if we take $\sigma$ and $p$ such that $3^{p-1}\left(\sigma_{1}+\sigma_{2}\right)<\pi^{2}$, we get that all mild solutions to our problem are globally exponentially asymptotically stable in the $p$-th mean, and theorem 4.1 (or theorem 4.2) also holds.

Now, assume that $\rho(t)=t=\tau(t), t \geq 0$, that is, we consider the problem without delays. In this case, we have $h=0, k=0, \sigma_{1}=\pi^{-2}, \sigma_{2}=\sigma^{2}$ and, we can get exponential stability in mean square $(p=2)$, and pathwise exponential stability, when $3\left(\pi^{-2}+\sigma^{2}\right)<\pi^{2}$ or, in other words, if $\sigma$ is such that $\sigma^{2}<\sigma_{0}^{2}=$ $\left(\pi^{2} / 3\right)-\left(1 / \pi^{2}\right) \simeq 3.1885$.

However, applying the results from Ichikawa [11] (see Example 2 from [11] for a similar problem), we could obtain exponential asymptotic stability in the $p$-th mean if

$$
p<1+\frac{2}{\sigma^{2}},
$$

i.e., if $\sigma^{2}<2 /(p-1)$. Therefore, if $2 \leq \sigma^{2}<\sigma_{0}^{2}$, Ichikawa's results do not even guarantee exponential stability in mean square $(p=2)$. 


\section{APPENDIX}

Now, we shall prove that there exists a unique mild solution to (1) under the hypotheses made in Section 2, although one can extend the result to a more general situation. We need the following additional assumption:

There exists a positive constant $D$ such that

$$
\|B(x)\|_{H}^{2}+\|C(x)\|_{2}^{2} \leq D^{2}\left(1+\|x\|_{H}^{2}\right) \quad \forall x \in H .
$$

TheOREM 1 In addition to (30), assume hypotheses in Section 2. Then, for each initial datum $\psi$ and every $T>0$, there exists a unique $H$-valued, $\mathcal{F}_{t}$-adapted (with $\mathcal{F}_{t}=\mathcal{F}_{0}$ for $\left.t<0\right)$ and predictable process $x(t) \quad(t \in[-h, T])$ satisfying (12)-(13) and $\int_{-h}^{T}\|x(t)\|_{H}^{2} d t<\infty \quad$ w.p.1.

Proof. We first prove uniqueness. We show that if $x_{1}(t), x_{2}(t)$ are two processes satisfying (12)-(13) then, for arbitrary $t \in[-h, T]$, it follows that $x_{1}(t)=x_{2}(t)$ w.p.1.

Set $x(t)=x_{1}(t)-x_{2}(t)$, then

$$
\begin{aligned}
x(t)= & \int_{0}^{t} S_{t-s}\left(B\left(x_{1}(\rho(s))\right)-B\left(x_{2}(\rho(s))\right)\right) d s \\
& +\int_{0}^{t} S_{t-s}\left(C\left(x_{1}(\tau(s))\right)-C\left(x_{2}(\tau(s))\right)\right) d w(s) .
\end{aligned}
$$

Define, for $i=1,2$ and $N \geq 0$,

$$
x_{i}^{N}(t)= \begin{cases}\psi(t) & \text { if } t<0 \\ x_{i}(t) & \text { if } \int_{0}^{t}\|x(s)\|^{2} d s \leq N \\ 0 & \text { if } \int_{0}^{t}\|x(s)\|^{2} d s>N\end{cases}
$$

and let $x^{N}(t)=x_{1}^{N}(t)-x_{2}^{N}(t)$. Since $\rho(t) \leq t$ and $\tau(t) \leq t$, we get that $x_{i}^{N}(\rho(t))=$ $x_{i}\left(\rho(t)\right.$ and $x_{i}^{N}(\tau(t))=x_{i}\left(\tau(t) \quad i=1,2\right.$, if $\int_{0}^{t}\|x(s)\|^{2} d s \leq N$.

Therefore, for $t \geq 0$

$$
\begin{aligned}
\left\|x^{N}(t)\right\|^{2} \leq & 2\left\|\int_{0}^{t} S_{t-s}\left(B\left(x_{1}^{N}(\rho(s))\right)-B\left(x_{2}^{N}(\rho(s))\right)\right) d s\right\|_{H}^{2} \\
& +2\left\|\int_{0}^{t} S_{t-s}\left(C\left(x_{1}^{N}(\tau(s))\right)-C\left(x_{2}^{N}(\tau(s))\right)\right) d w(s)\right\|_{H}^{2}
\end{aligned}
$$

and,

$$
\begin{aligned}
E\left\|x^{N}(t)\right\|^{2} \leq & 2 E \| \int_{0}^{t} S_{t-s}\left(B\left(x_{1}^{N}(\rho(s))\right)-\left(B\left(x_{2}^{N}(\rho(s))\right)\right) d s \|_{H}^{2}\right. \\
& +2 E \| \int_{0}^{t} S_{t-s}\left(C\left(x_{1}^{N}(\tau(s))\right)-\left(C\left(x_{2}^{N}(\tau(s))\right)\right) d W(s) \|_{H}^{2}\right. \\
= & 2 I_{1}+2 I_{2} .
\end{aligned}
$$


Next, we estimate $I_{1}$ and $I_{2}$ by using (18) for $p=2$ :

$$
\begin{aligned}
I_{1} & \leq E\left[\int_{0}^{t}\left\|S_{t-s}\right\|\left\|B\left(x_{1}^{N}(\rho(s))\right)-B\left(x_{2}^{N}(\rho(s))\right)\right\| d s\right]^{2} \\
& \leq M^{2} E\left[T \int_{0}^{t}\left\|B\left(x_{1}^{N}(\rho(s))\right)-B\left(x_{2}^{N}(\rho(s))\right)\right\|^{2} d s\right] \\
& \leq M^{2} T L_{1}^{2} \int_{0}^{t} E\left\|x_{1}^{N}(\rho(s))-x_{2}^{N}(\rho(s))\right\|^{2} d s \\
& \leq M^{2} T L_{1}^{2} \int_{\rho(0)}^{\rho(t)} E\left\|x_{1}^{N}(u)-x_{2}^{N}(u)\right\|^{2} d u \\
& \leq M^{2} T L_{1}^{2} \int_{0}^{t} E\left\|x^{N}(s)\right\|^{2} d s, \\
I_{2} & \leq M^{2} \int_{0}^{t} E\left\|C\left(x_{1}^{N}(\tau(s))\right)-C\left(x_{2}^{N}(\tau(s))\right)\right\|_{2}^{2} d s \\
& \leq M^{2} L_{2}^{2} \int_{0}^{t} E\left\|x^{N}(s)\right\|^{2} d s .
\end{aligned}
$$

Hence,

$$
E\left\|x^{N}(t)\right\|^{2} \leq 2 M^{2}\left(T L_{1}^{2}+L_{2}^{2}\right) \int_{0}^{t} E\left\|x^{N}(s)\right\|^{2} d s .
$$

Gronwall's inequality implies that $E\left\|x^{N}(t)\right\|^{2}=0$ for all $t$. Thus $x^{N}(t)=0$. Since $x_{i}^{N}(t) \rightarrow x_{i}(t)$ w.p.1, $x^{N}(t) \rightarrow x(t)$ w.p.1, from which follows $x(t)=0$ w.p.1.

Finally, we prove existence. As $\rho^{\prime}(t) \geq 1$ and $\rho(t) \leq t$, then $\rho(t)-t$ is nondecreasing and nonpositive, so there exist only three posibilities:

$(\rho .1) \lim _{t \rightarrow \infty}(\rho(t)-t)=-\rho_{*}<0$, which implies $\rho(t)<t-\rho_{*}$ for $0 \leq t \leq \rho_{*}$.

$(\rho .2) \lim _{t \rightarrow \infty}(\rho(t)-t)=0$, but $\rho(t)<t$ for any $t \geq 0$, which allows us to take an increasing sequence $\left\{t_{k}\right\}_{k \geq 0}$ such that $t_{0}=0, t_{k} \uparrow \infty$ and $\rho\left(t_{k+1}\right) \leq t_{k}$.

( $\rho .3)$ There exists $T_{\rho}>0$ such that $\rho(t)<t$ for $t<T_{\rho}$, but $\rho(t)=t$ for $t \geq T_{\rho}$.

But, as $\tau(t)$ satisfies the same hypotheses, we have analogous posibilities $(\tau .1),(\tau .2),(\tau .3)$. Therefore, combining these, we consider the following six different cases:

i) Assume $(\rho .1),(\tau .1)$. Let $\delta=\min \left\{\rho_{*}, \tau_{*}\right\}$ and consider the problem on $[0, \delta]$. Since $\rho(t) \leq t-\delta$ and $\tau(t) \leq t-\delta$,

$$
x(t)=S_{t} \psi(0)+\int_{0}^{t} S_{t-s} B(\psi(\rho(s))) d s+\int_{0}^{t} S_{t-s} C(\psi(\tau(s))) d w(s)
$$

is the mild solution to (1) on the interval $[0, \delta]$. By induction, our problem can be solved on $[k \delta,(k+1) \delta]$ for all $k \geq 0$ and therefore on $[0, \infty)$. 
ii) Assume $(\rho .2),(\tau .2)$. In this case, we can choose an increasing sequence $\left\{t_{k}\right\}_{k \geq 0}$ such that $t_{0}=0, t_{k} \uparrow \infty$ and $\rho\left(t_{k+1}\right) \leq t_{k}, \tau\left(t_{k+1}\right) \leq t_{k}$. So, we can construct the mild solution on $\left[t_{k}, t_{k+1}\right]$ for all $k \geq 0$ as in Case i).

iii) Assume $(\rho .3),(\tau .3)$. If $T_{\rho} \neq T_{\tau}$, suppose $T_{\rho}<T_{\tau}$. Then we proceed on $\left[0, T_{\rho}\right)$ in the same way as Case ii). Since it is not difficult to show that $x(t)$ converges to $x\left(T_{\rho}\right)$ in mean square (when $t \rightarrow T_{\rho}$ ), the problem on $\left[T_{\rho}, T_{\tau}\right.$ ) can be equivalently stated as

$$
x(t)=S_{t-T_{\rho}} x\left(T_{\rho}\right)+\int_{T_{\rho}}^{t} S_{t-s} B(x(s)) d s+\int_{T_{\rho}}^{t} S_{t-s} C(x(\tau(s))) d w(s)
$$

and the existence of mild solution is guaranteed by using the results on existence of mild solution for the nondelayed equation (see Da Prato and Zabczyk [7], for instance) and the method of Case ii). Next, on $\left[T_{\tau}, \infty\right)$ the equation for mild solutions is

$$
x(t)=S_{t-T_{\tau}} x\left(T_{\tau}\right)+\int_{T_{\tau}}^{t} S_{t-s} B(x(s)) d s+\int_{T_{\tau}}^{t} S_{t-s} C(x(s)) d w(s)
$$

which is an equation without delays and, consequently, the existence of solution is well known.

iv) Assume $(\rho .1),(\tau .2)$. Then, we have that $\rho(t)<t-\rho_{*}$ and $\tau(t)<t$ for all $t$. Denoting by $n_{0}$ the natural number such that $t_{n_{0}} \leq \rho_{*} \leq t_{n_{0}+1}$, we solve the equation on $\left[0, \rho_{*}\right]$ as in Case ii), and by induction, on $\left[k \rho_{*},(k+1) \rho_{*}\right]$ for all $k \geq 0$.

v) Assume $(\rho .1),(\tau .3)$. In this case, $\rho(t)<t-\rho_{*}$ and $\tau(t)<t$ for $t<T_{\tau}$ and $\tau(t)=t$ for all $t_{\tau}$. Thus, on the interval $\left[0, T_{\tau}\right)$ we can argue as in Case iv) and, on $\left[T_{\tau}, \infty\right)$ as in Case i) but, with only one delayed term (see Case iii), for a similar situation).

vi) Assume $(\rho .2),(\tau .3)$. It follows that $\rho(t)<t$ for all $t$, and $\tau(t)<t$ for all $t<T_{\tau}, \tau(t)=t$ for all $t \geq T_{\tau}$. The existence of the mild solution on $\left[0, T_{\tau}\right)$ is showed as in Case ii). On $\left[T_{\tau}, \infty\right)$, the arguments of Case ii) for the equation

$$
x(t)=S_{t-T_{\tau}} x\left(T_{\tau}\right)+\int_{T_{\tau}}^{t} S_{t-s} B(x(\rho(s))) d s+\int_{T_{\tau}}^{t} S_{t-s} C(x(s)) d w(s)
$$

can be used.

\section{ACKNOWLEDGEMENTS}

The authors wish express their thanks to Prof. J. Real and J.A. Langa for their useful and interesting suggestions on the topic of this paper.

This work has been partially supported by D.G.I.C.Y.T. (Spain) Proyectos PB920696 and PB95-1242. 


\section{References}

[1] T. Caraballo, Algunos resultados de estabilidad para ecuaciones en derivadas parciales estocásticas con retardo, Tesis Doctoral, Univ. Sevilla (1988)

[2] T. Caraballo, Asymptotic Exponential Stability of Stochastic Partial Differential Equations with Delay, Stochastics and Stochastics Reports, $\underline{33}$ (1990), $27-47$.

[3] T. Caraballo and J. Real, Partial Differential Equations with Delayed Random Perturbations: Existence, Uniqueness, and Stability of Solutions, Stoch. Anal. Appl., 11 (5) (1993), 497-511.

[4] T. Caraballo and J. Real, On The Pathwise Exponential Stability of Non-Linear Stochastic Partial Differential Equations, Stoch. Anal. Appl., 12 (5) (1994), 517-525.

[5] P. L. Chow, Stability of Nonlinear Stochastic-Evolution Equation, J. Math. Anal. Appl., $\underline{89}$ (2) (1982), 400-419.

[6] R.F. Curtain, Stability of Stochastic Partial Differential Equation, J. Math. Anal. Appl., $\underline{79}$ (2) (1981), 352-369.

[7] G. Da Prato and J. Zabczyk, Stochastic Equations in Infinite Dimensions, Cambridge University Press, Cambridge, 1992.

[8] G. Da Prato and J. Zabczyk, A note on stochastic convolution, Stoch. Anal. Appl., 10 (2) (1992), 143-153.

[9] L.E. El'SGOL'Ts And S.B. Norkin, Introduction to the Theory and Applications of Differential Equations with Deviating Arguments, Academic Press, London, 1973.

[10] U. G. Haussmann, Asymptotic Stability of the Linear Itô Equation in InfiniteDimension, J. Math. Anal. Appl., $\underline{65}$ (1978), 219-235.

[11] A. Ichikawa, Stability of Semilinear Stochastic Evolution Equations, J. Math. Anal. Appl., 90 (1982), 12-44.

[12] N.N. Krasovskit, Stability of motions, Stanford University Press, 1963. 
[13] H.J. Kushner, On the Stability of Process Defined by Stochastic DifferenceDifferential Equations, J. Differential Equations, 4 (1968), 424-443.

[14] G.S. Ladde, V. Lakshmikantham and B.G. Zhang, Oscillation theory of differential equations with deviating arguments, Marcel Dekker, New York, 1987.

[15] X. MAO, Exponential stability for stochastic differential delay equations in Hilbert spaces, Quart. J. Math., $\underline{42}$ (2) (1991), 77-85.

[16] J. REAL, Stochastic partial differential equations with delays, Stochastics, $\underline{8}$ (2) (1982-83), 81-102.

[17] J. ZABCZYK, On stability of infinite dimensional stochastic systems, Probability Theory, Z. Ciesislki (Ed.), Banach Center Publications, vol. $\underline{5}$ (1979), 273-281.

[18] J. ZABCZYK, Linear stochastic systems in Hilbert spaces: structural properties and limit behaviour, Banach Center Publications, vol. 14, Warswa, 1985. 